\title{
Representaçóes sociais da sistematização da assistência de enfermagem sob a ótica de enfermeiros
}

Nurses' social representations of nursing care systematization

Representaciones sociales de la sistematización de la asistencia de enfermería desde la perspectiva de los enfermeros

Ana Caroline da Costa*; José Vitor da Silva**

\section{Resumo}

Enquadramento: A sistematização da assistência de enfermagem (SAE) é uma metodologia com sólida estrutura teórica e científica, para planear, organizar e sistematizar os cuidados.

Objetivos: Conhecer os significados da SAE sob a ótica dos enfermeiros de uma instituiçáo hospitalar da cidade de Itajubá, Minas Gerais - Brasil.

Metodologia: Estudo de abordagem qualitativa, do tipo exploratório e descritivo. Amostra intencional de 30 enfermeiros. Para a análise dos dados, utilizou-se o método do discurso do sujeito coletivo.

Resultados: Do tema, significados da SAE, emergiram as seguintes representaçóes sociais: estratégia que norteia o cuidado; atividade exclusiva do enfermeiro; e é algo que não funciona na prática.

Conclusáo: Apesar da SAE ser amplamente discutida no âmbito da formaçáo e exercício profissional, ainda existe a representação de ser um procedimento desacreditado na sua prática, embora seja reconhecida como norteadora dos cuidados e exclusiva do enfermeiro.

Palavras-chave: enfermagem; metodologia; cuidados de enfermagem

\section{Abstract}

Background: Nursing care systematization (NCS) is a methodology based on a solid theoretical and scientific framework that allows planning, organizing, and systematizing care.

Objectives: To identify the meanings that nurses assign to NCS in a hospital located in the city of Itajubá, Minas Gerais - Brazil.

Methodology: This was an exploratory and descriptive study with a qualitative approach using a purposive sample of 30 nurses. Data were analyzed using the discourse of the collective subject method.

Results: The following central ideas emerged as meanings assigned to NCS: it is a care guiding strategy; it is an activity that is carried out exclusively by nurses; and it does not work in practice.

Conclusion: Although NCS is a widely discussed topic in professional training and practice, its implementation in clinical practice is still questioned. However, NCS is recognized as a care guiding strategy and a nursing activity.

Keywords: nursing; methodology; nursing care
*RN., Enfermeira Especialista, Professora, Faculdade Wenceslau Braz, 84950-000 Itajubá, MG, Brasil [carolinea16@yahoo.com.br]. Contribuição no artigo: pesquisa bibliográfica, recolheu dados, análise e avaliações dos dados, discussão e escrita do artigo. Morada para correspondência: Rua Nossa Senhora - casa 33 B - Bairro São Vicente, 37502-074, Itajubá, Brasil

**Ph.D., Professor, Faculdade Wenceslau Braz, 84950-000, Itajubá, MG, Brasil [enfjvitorsilva@oi.com.br]. Contribuição no artigo pesquisa bibliográfica, análise e avaliações dos dados, discussão e escrita do artigo.

\section{Resumen}

Marco contextual: La sistematización de la asistencia de enfermería (SAE) es una metodología con una estructura teórica y científica sólida para planear, organizar y sistematizar los cuidados.

Objetivos: Conocer los significados de la SAE desde la perspectiva de los enfermeros de una institución hospitalaria de la ciudad de Itajubá, Minas Gerais - Brasil. Metodología: Estudio de enfoque cualitativo, exploratorio y descriptivo. Muestra intencional de 30 enfermeros. Para el análisis de los datos, se utilizó el método del discurso del sujeto colectivo.

Resultados: Del tema significados de la SAE surgieron las siguientes representaciones sociales: estrategia que orienta los cuidados; actividad exclusiva del enfermero; y es algo que no funciona en la práctica.

Conclusión: Aunque la SAE se discute bastante en el ámbito de la formación y el desempeño profesional, todavía existe la representación de que es un procedimiento desacreditado en su práctica, aunque se la reconoce como orientadora de los cuidados y exclusiva del enfermero.

Palabras clave: enfermería; metodología; atención de enfermería 


\section{Introdução}

A sistematização da assistência de enfermagem (SAE) configura-se como uma metodologia com sólida estrutura teórica e científica, para planear, organizar e sistematizar os cuidados. A SAE tem como objetivos perceber as situações de saúde-doença e as necessidades de cuidados de enfermagem, bem como auxiliar as intervençôes de promoção, prevenção, recuperação e reabilitação da saúde do indivíduo, família e comunidade, além de promover maior segurança e qualidade durante a assistência prestada (Chaves, Silva, Motta, Ribeiro, \& Andrade, 2016). Desta forma, a SAE é utilizada como uma metodologia científica que viabiliza o trabalho do enfermeiro, permitindo que alcance resultados pelos quais ele é responsável (Truppel, Meier, Calixto, Peruzzo, \& Crozeta, 2009).

Contudo, a implementação da SAE, na maioria dos serviços do Brasil, permanece muito aquém do que está especificado na legislação de enfermagem, apesar de ser amplamente discutida e utilizada no contexto académico. Mesmo sendo obrigatória a sua utilização, ainda não faz parte do trabalho diário do enfermeiro, muitas vezes, devido à sobrecarga de trabalho imposta e ao número reduzido de enfermeiros, que acabam por dar prioridades às atividades burocráticas e administrativas, que também fazem parte das suas atribuições enquanto profissionais (Nery, Santos, \& Sampaio, 2013). Acrescenta-se a isto, a falta de paradigma do enfermeiro.

Muitas são as dificuldades registadas que podem estar a contribuir para a ineficiência da aplicação total da SAE no dia-a-dia da assistência de enfermagem. Alguns autores apontam que as dificuldades para a implementação da SAE não se restringem apenas à prática profissional, mas parecem iniciar-se desde o ensino académico, quando há dificuldades, por parte dos docentes, de desenvolverem estratégias didáticas padronizadas, adequadas e eficientes para a aprendizagem efetiva dessa metodologia de assistência (Cavalcante et al., 2011).

Assim, este estudo reafirma a necessidade da implementação da SAE como ferramenta norteadora para as práticas de enfermagem, permitindo aos enfermeiros o reconhecimen- to da assistência prestada, organização no trabalho, autonomia para tomada de decisóes e, sobretudo, a qualidade assistencial, focada no cuidado integral e individual.

Fornecerá e fortalecerá, também, dados importantes para novos estudos em outras realidades, despertando o interesse para a implementação da SAE nas diversas instituições de saúde, e, desta forma, permitindo cuidados qualificados aos pacientes de forma sistemática e com cientificidade. Desta forma, este estudo procurou conhecer os significados da SAE sob a ótica dos enfermeiros de uma instituição hospitalar da cidade de Itajubá, Minas Gerais - Brasil.

\section{Enquadramento}

A agregação de novos conhecimentos observados durante a prática do cuidar de enfermagem proporcionou a elaboração das teorias de enfermagem, conferindo à prática em enfermagem um caráter científico. A elaboração de postulados teóricos foi a alternativa encontrada para compilar todo o conhecimento da enfermagem, contribuindo para a assistência baseada não somente na dimensão biológica do ser humano, mas também na compreensão do indivíduo como ser psico-sócio-espiritual (Chaves et al., 2016).

As primeiras tentativas de sistematizar os cuidados de enfermagem no Brasil foram propostas por Horta, na década de 1970. Wanda de Aguiar Horta estudou o processo de enfermagem (PE) em profundidade e as suas reflexões culminaram na Teoria das Necessidades Humanas Básicas, publicada em 1979. A proposta dessa enfermeira para o PE é seguida em todo o Brasil até aos dias atuais, na maioria das unidades de saúde. A sua proposta metodológica estabelece o PE realizado em seis etapas: histórico de enfermagem, diagnóstico de enfermagem, plano assistencial, prescrição de enfermagem, evolução de enfermagem e prognóstico de enfermagem (Garcia, 2016). Sendo assim, a SAE surgiu quando a enfermagem começou a sistematizar as suas ações, com o intuito de integrar o seu conhecimento teórico à prática assistencial e, assim, construir o corpo de conhecimento próprio na busca de consoli- 
dação da profissão enquanto ciência. (Varela \& Fernandes, 2013, p. 125)

No que tange aos aspetos legais, a resolução 358/2009 do Conselho Federal de Enfermagem (COFEN), dispóe sobre a SAE e a implementação do $\mathrm{PE}$ em ambientes públicos ou privados em que ocorrem os cuidados de enfermagem. A SAE é uma atividade exclusiva do enfermeiro, utiliza métodos e estratégias de trabalho científico para a identificação das situações de saúde/doença, subsidiando ações de assistência de enfermagem que possam contribuir para a promoção, prevenção, recuperação e reabilitaçâo da saúde, tanto na dimensão individual, como no contexto familiar e comunidade onde estão inseridos (Carvalho \& Barcelos, 2017; Oliveira, Coelho, Almeida, Lisboa, \& Macêdo, 2012).

A SAE deve ser realizada em cinco etapas inter-relacionadas, interdependentes e recorrentes, sendo: histórico de enfermagem, diagnóstico de enfermagem, planeamento de enfermagem, implementação de enfermagem e avaliação de enfermagem. Assim, incorporar a SAE na assistência é uma forma de tornar a enfermagem com mais embasamento científico, promovendo cuidados de enfermagem individualizados, humanizados, contínuos, mais justos e com qualidade para a paciente (Carvalho \& Barcelos, 2017; Chaves et al., 2016; Nery et al., 2013).

A SAE é operacionalizada por meio do PE que é um instrumento metodológico e sistemático de prestação de cuidados, constituído por etapas inter-relacionadas e organizadas por fases. Estas fases proporcionam ao enfermeiro uma visão integral do paciente, além de auxiliar na tomada de decisão para execução de cuidados baseados em evidência (Carvalho \& Barcelos, 2017; Chaves et al., 2016).

A implementação e operacionalização da SAE exige dos enfermeiros inúmeros conhecimentos. Sendo assim, torna-se fundamental o envolvimento de toda equipa de enfermagem, a fim de compartilhar e disseminar os saberes, por meio de grupos de estudos, discussóes e processos de educação permanente em serviço (Benedet, Gelbcke, Amante, Padilha, \& Pires, 2016; Massaroli et al., 2015).

Desde os primeiros estudos sobre o PE e a SAE, que se têm vindo a tentar implementar estas metodologias de trabalho. Apesar da re- solução n 358/2009 da COFEN estar vigente, ela por si só não oferece o apoio necessário à implementação, uma vez que há inúmeras dificuldades vivenciadas na prática do enfermeiro. Grande parte dos desafios enfrentados pelos enfermeiros na tentativa de operacionalizar a SAE é a falta de fundamentação teórica científica e a sobrecarga de trabalho (Carvalho \& Barcelos, 2017).

\section{Questáo de Investigaçáo}

Quais são as representaçóes sociais dos enfermeiros de uma instituição hospitalar sobre a sistematização da assistência de enfermagem?

\section{Metodologia}

Trata-se de um estudo de abordagem qualitativa, do tipo exploratório e descritivo, desenvolvido num hospital-escola, no município de Itajubá, Minas Gerais - Brasil. A amostra foi constituída por 30 enfermeiros e a amostragem foi intencional ou teórica. Os critérios de elegibilidade foram: ser enfermeiro e fazer parte do quadro de profissionais desse hospital-escola.

Para a colheita dos dados, foi utilizado um roteiro de entrevista semiestruturada com a seguinte pergunta: "Para você, o que significa sistematização da assistência de enfermagem?".

As respostas foram gravadas sob prévia permissão dos participantes, com posterior transcrição de falas, garantindo a fidedignidade dos relatos. A entrevista ocorreu em local adequado, longe de ruídos e individualmente. Foram esclarecidas todas as dúvidas antes de se iniciar a gravação. Ocorreu o anonimato dos enfermeiros, denominando-os com a letra $\mathrm{E}$ (de enfermeiro), seguida de numeração ordinal conforme a realização das entrevistas (E1, E2, E3...).

Os dados foram analisados e interpretados utilizando-se o método do discurso do sujeito coletivo (DSC), que é uma proposta de organização e tabulação de dados qualitativos, de natureza verbal, obtidos de depoimentos, artigos de jornal e revistas. É uma forma de fazer a coletividade falar 
diretamente (Lefèvre \& Lefèvre, 2010). Foram utilizadas deste método três figuras metodológicas: 1) Expressóes-chave (ECH), que envolvem transcriçôes literais das respostas gravadas; 2) Ideias centrais (IC), que são um nome ou expressão linguística que descreve, de maneira precisa e fidedigna, o sentido de cada discurso analisado e 3) DSC, que é um discurso-síntese redigido na primeira pessoa do singular e composto pelas $\mathrm{ECH}$ que têm a mesma IC (Lefèvre \& Lefèvre, 2010). O desenvolvimento do método para a obtenção das representações sociais dos enfermeiros consistiu nos seguintes passos, segundo Lefèvre \& Lefèvre, (2010): Copiou-se na íntegra o conteúdo de todas as respostas obtidas por meio do roteiro de entrevista semiestrutura$\mathrm{da}$; agruparam-se as IC equivalentes (aquelas com o mesmo sentido), e de seguida, foi construído um DSC para cada agrupamento das ICs.

Este estudo obteve aprovação da Comissão de Ética em Pesquisa mediante Parecer Consubstanciado no 470.795, Certificado CAAE 25077713.3.0000.5559.

\section{Resultados e discussáo}

Do tema significados da SAE emergiram as seguintes ideias centrais: estratégia que norteia o cuidado; atividade exclusiva do enfermeiro; e é algo que não funciona na prática.

A primeira ideia central - Estratégia que norteia o cuidado - fornece direção na prática de trabalho, auxilia na organização e no planeamento da assistência prestada, favorecendo melhor resultado na implementação do plano de cuidados. Dessa maneira, o enfermeiro sente-se mais seguro durante a prestação dos cuidados, pois a SAE também permite uma conduta mais fundamentada, ancorada em conhecimento científico. Isto contribui para que o profissional deixe de atuar de forma intuitiva ou impulsiva, só cumprindo tarefas, como, na maioria das vezes, é visto. Com a $\mathrm{SAE}$, o enfermeiro adquire a autonomia para a tomada de decisão, construindo assim o seu próprio conhecimento e contribuindo cada vez mais para consolidar a enfermagem como ciência.

Este método exige do enfermeiro pensamento crítico, e requer constante atualização, experiências e habilidades para exercer a profissão com autonomia baseada nos conhecimentos técnico-científicos (Garcia, 2016; Silva, Oliveira, Neves, \& Guimarães, 2011).

Corrobora com os aspetos expostos acima, o seguinte DSC:

a SAE é uma metodologia que norteia a assistência de enfermagem, tornando-a mais científica, tendo melhores resultados nos cuidados. É uma maneira de a enfermeira organizar, planejar, colher resultados da assistência. A SAE norteia quanto uma melhor qualidade no cuidado ao paciente, o que nos ajuda a ter um planejamento perante as situaçóes adversas do dia a dia. É a assistência de enfermagem organizada dentro de um roteiro, uma sequência, uma padronização para se dar a assistência melhor ao nosso cliente. A SAE organiza a assistência de enfermagem quando se realizam todas suas etapas, dando autonomia para o enfermeiro tomar as decisôes necessárias para o cuidado do paciente. (E1, E2, E4, E10, E19, E20, E28, Fev. 2014)

Pautada no discurso acima, a SAE pode ser entendida como uma metodologia que favorece e direciona os cuidados de enfermagem, além de fornecer as ferramentas necessárias para a organização do trabalho do enfermeiro (Benedet et al., 2016; Varela \& Fernandes, 2013). Silva et al. (2011) mostram que a SAE permite organizar e padronizar a prática profissional, além de constituir um instrumento para gerir e otimizar a assistência de enfermagem de forma organizada, segura, dinâmica e competente.

Apesar de existirem muitas críticas referentes à sua forma de utilização, essa metodologia tem sido considerada como um provedor da uniformização da linguagem, por sistematizar a prática e ampliar a autonomia profissional (Cogo et al., 2012).

Resultados de estudos também mencionam que, com a utilização da SAE, os enfermeiros reconhecem que o foco da assistência deve ser o cliente. Mediante a sua aplicação, obtém-se a identificação dos problemas e necessidades, promove-se o planeamento e a organização e fundamentação dos cuidados, bem como 
facilita a prática assistencial do enfermeiro (Medeiros, Santos, \& Cabral, 2012; Menezes, Priel, \& Pereira, 2011).

Como método de trabalho, a SAE exige do enfermeiro um pensamento crítico ou reflexivo, raciocínio individualizado e dimensionamento adequado da equipa. $\mathrm{O}$ enfoque integral ajuda a assegurar que as intervenções sejam feitas para o indivíduo e não para a doença (Medeiros et al., 2012; Menezes et al., 2011).

Com a utilização da SAE, há uma melhora significativa da qualidade da assistência, a profissão ganha cientificidade, além de garantir vantagens, como promoção da integração entre a equipa de enfermagem e demais membros da equipa de saúde, pacientes e familiares (Oliveira et al., 2012).

Em relação à segunda IC - Atividade exclusiva do enfermeiro, ressalta-se que algumas funções de natureza profissional são específicas do enfermeiro, não podendo ser delegadas a outros. O COFEN esclarece na sua resolução no 272/2002, revogada pela resoluçáo no $358 / 2009$ da COFEN, que a SAE é uma atividade exclusiva do enfermeiro, cabendo a ele a sua implementação nas práticas de trabalho (Carvalho \& Barcelos, 2017).

Entretanto, observa-se ainda falta de consciencialização para o fortalecimento da implementação da SAE, pois são poucas as instituições que fazem uso dessa metodologia. Fortalecendo os fatos acima, temos o seguinte DSC:

a SAE é uma ação exclusiva do enfermeiro, deve ser realizada na admissão. É constituída por cinco etapas: histórico de enfermagem, diagnóstico, planeamento, implementação e evolução ou avaliação de enfermagem. É uma atividade exclusiva do enfermeiro que utiliza um método de trabalho ordenado para subsidiar as açóes de assistência de enfermagem. Tem que ser realizada pelo enfermeiro e auxilia no cuidado, priorizando a assistência conforme a gravidade do paciente. Por meio dela se realiza a identificação das situaçóes de saúde/doença propiciando a prescrição e assistência de enfermagem. (E9, E13, E15, E25, E27, Fev. 2014)

Nesse sentido, a Lei No 7.498/86 do Exercício Profissional define como atividades exclusivas do enfermeiro a consulta de enfermagem e a prescrição da assistência de enfermagem (Moreira, Santos, Oliveira, Reis, \& Lima, 2013). Por ser uma ação exclusiva do enfermeiro, a SAE exige a utilização de conhecimentos técnico-científicos e humanos na assistência ao paciente para caracterizar a sua prática profissional, colaborando na definição do seu papel. Para promover os cuidados e reestabelecer as necessidades do paciente, o enfermeiro precisa de desenvolver as fases do PE, pautado num referencial teórico (Pains, Oliveira, Moreira, \& Malta, 2009).

A terceira IC evidenciou-se pela seguinte representação: a SAE é vista como algo que não funciona na prática. Mesmo havendo uma obrigatoriedade legal em relação ao seu uso, e com diversas pesquisas acerca da SAE, fundamentando a sua importância na prática diária, alguns enfermeiros não conseguem implementá-la no seu trabalho, não compreendem o seu significado, atribuindo-lhe o significado de perda de tempo, que não ajudará no processo de cuidar. Esse facto, muitas vezes, é justificado pela falta de tempo do enfermeiro.

$\mathrm{O}$ envolvimento do enfermeiro com burocracias e diversas funçôes complementares não favorece a dedicação à realizaçãao da SAE. Vale salientar também que a falta de ideologia, valor, interesse e empenho do próprio enfermeiro em planear e executar as etapas da SAE pode ser um fator contribuinte para a não efetivação dessa metodologia.

Estudos apontam que uma das dificuldades para a implementação da SAE é a falta de preparação ou de capacitação do profissional e falha da instituição em capacitar os enfermeiros (Santos \& Silva, 2013). Acrescentam-se também outros fatores contribuintes para a falta de adoção da SAE nas instituiçóes, que são: falta de planeamento do tempo do enfermeiro e a sua demanda de trabalho, a pouca valorização cultural da SAE, falta de hábito e dificuldade para aquisição de habilidade, além da resistência de se trabalhar com novas metodologias (Oliveira et al., 2012). Evidenciando o que se mencionou anteriormente, regista-se, a seguir, o seguinte DSC:

dentro da nossa realidade, a SAE é uma perda de tempo, pois não temos tempo hábil para sua execução e muito menos para os servidores executarem; faltam técnicos e enfermeiras também, pois 
ela [enfermeira] acumula funçôes, faz serviço de farmacêutico, manutenção, não tendo tempo para evoluir. Aqui, a enfermeira é voltada para a burocracia, aí os técnicos acabam ocupando o lugar. Na teoria é muito bonito, porém na prática deixa muito a desejar. As etapas não são realizadas de forma adequada, ou mesmo não são realizadas de forma alguma. Se a SAE fosse realmente feita de forma correta, ajudaria bastante, o que não é nossa realidade, infelizmente. (E29, E30, Fev. 2014).

Por meio do depoimento dos participantes acima constatou-se que eles só percebem a eficácia da SAE na teoria, e que se ela fosse aplicada na prática de forma correta, facilitaria muito a assistência de enfermagem. Porém, apontam várias dificuldades que impedem a sua implementação, como: falta de tempo, número reduzido de funcionários e excesso de burocracia impossibilitam o enfermeiro de executar as etapas da SAE.

Estudos sobre a perceção dos profissionais quanto à importância da SAE, mostraram que $75 \%$ dos enfermeiros a consideravam muito importante. Entretanto, 74\% responderam que estavam desmotivados para executá-la. Diversos foram os motivos alegados para não trabalharem com a sistematização. Dentre eles, destacaram-se: reduzido número de profissionais/sobrecarga de trabalho/elevado número de pacientes (54\%); condiçôes inadequadas do serviço (13\%); burocracia (4\%), representando $67 \%$ de problemas relacionados a condiçóes de trabalho (Silva et al., 2011).

Esses fatores impeditivos de aplicação da SAE também são citados, incluindo a falta de motivação entre os profissionais, falta de empenho das políticas institucionais, que dificultam a sua implementação, falta de conhecimento, pouca ou falta de prática dos profissionais, dificuldade em entender o diagnóstico de enfermagem e aplicá-lo na prática, preocupação excessiva com a demanda do serviço e náo com qualidade da assistência e inexistência de formulários para realização da SAE (Silva \& Carvalho Filha, 2017). Todas essas limitaçóes foram citadas no discurso acima, evidenciando que são problemas frequentes de diversas instituiçóes e que comprometem significativamente a adesão à SAE.
Outros estudos também ressaltam alguns desafios que fazem parte da trajetória de implementação da SAE nas instituiçôes e que podem ajudar na sua efetivação, a saber: o conhecimento, o número de enfermeiros nos serviços, o envolvimento deles com o processo, a valorização por parte da administração da instituição, bem como os indicadores de resultado da assistência. Ao mesmo tempo, realizar esse processo requer do profissional base científica, conhecimento, habilidades e atitudes pautadas no compromisso ético, responsabilidade e assumir o cuidar do outro (Monteiro, Monteiro, Araújo, Gouveia, \& Alencar, 2013).

Infelizmente, no contexto atual, ainda existem várias dificuldades para a execução da SAE, envolvendo não apenas a redução e carência de recursos humanos, mas a forma como o profissional se apropria do conhecimento sobre o tema (Carvalho \& Barcelos, 2017; Monteiro et al., 2013; ).

Mesmo com o empenho do Conselho COFEN e de toda a classe profissional, trata-se de um conhecimento que, apesar de ter sido introduzido no Brasil, na década de 1970, ainda apresenta uma enorme lacuna entre a produção do conhecimento e a sua aplicabilidade na prática diária do enfermeiro. (Silva et al., p. 1381)

A implementação da SAE vai além de uma sequência de passos a serem seguidos, exige do enfermeiro entendimento e familiaridade com os diagnósticos de enfermagem e raciocínio, julgamento clínico para adequar as necessidades do indivíduo às condiçôes de trabalho, tornando-as mais complexas do que menciona a teoria. (Silva et al., 2011, p. 1381)

Por outro lado, vários estudos contradizem o DSC descrito acima, revelando que a SAE constitui um importante instrumento de trabalho para os profissionais de enfermagem, pois é um método que oferece autonomia ao enfermeiro, além de avaliar a qualidade dos cuidados prestados ao paciente adequado às suas necessidades (Santos \& Silva, 2013).

Em relação à questão se o enfermeiro deveria trabalhar com a SAE, encontrou-se, num estudo realizado no Brasil, que a grande maio- 
ria, 92\%, concordava e, dentre os principais benefícios que justificariam a adesão ao método, estavam a melhorar a qualidade da assistência $(44 \%)$ e $18 \%$ relataram que ela promove autonomia ao profissional (Silva et al., 2011).

Portanto, justifica-se a importância da implementação da SAE no ambiente de trabalho do enfermeiro, seja na instituição hospitalar, privada ou pública, nos ambulatórios, clínicas, atenção básica e principalmente nas escolas de enfermagem, contribuindo assim para a aquisição das competências necessárias, habilidades e atitudes, na utilização deste método. Com isso, a profissão tornar-se-á mais científica, reconhecida e valorizada, além de permitir cuidados qualificados e, individualizados e integrados para o paciente, família e comunidade.

\section{Conclusão}

Considera-se que a SAE é uma necessidade no serviço de saúde, e que ainda não é um paradigma de enfermagem, considerando que há enfermeiros que ainda não despertaram para a sua essência, necessidade e importância na realização da assistência de enfermagem. Consequentemente, ainda existem lacunas, resistências e dificuldades na sua implementação.

Algumas falhas são decorrentes da própria característica da profissão de enfermagem, que, na maioria das vezes, trabalha com um número insuficiente de funcionários, alegando não ter tempo para a execução da metodologia. Porém, isto não se justifica totalmente, sendo que, com a funcionalidade da SAE, facilitaria ainda mais o trabalho do enfermeiro, pois trata-se de uma metodologia organizada, planeada e fundamentada em princípios científicos, facilitando a assistência de enfermagem.

Portanto, face às inúmeras dificuldades encontradas para implementação da SAE, cabe aos enfermeiros e também às instituições responsáveis, maior envolvimento, compromisso ético e profissional aliado ao conhecimento científico no sentido de procurar melhorias e valorização profissional. Falta ideologia do enfermeiro para conseguir implementar a SAE, pois infelizmente muitos ainda estão habitua- dos a atividades funcionais e rotineiras, sem se importarem em alcançar autonomia, além da falta de apoio institucional, pois não se trata do interesse de ambos.

Ao comparar os resultados deste estudo com outros já realizados, observou-se que estes são similares aos anteriores. Isto pode significar que a implementação da SAE não tem sofrido modificações ou alteraçóes. Não se tem avançado com a introdução de novos aspetos para a ampliação da sua ideologia na assistência de enfermagem, parecendo que não tem sido mais objeto de preocupação.

Sugere-se que, para obter mudanças nesses cenários, haja mudanças de paradigmas. É necessária a introdução de disciplinas que englobem a SAE nas graduaçóes em enfermagem, ministradas por docentes envolvidos e que acreditam que a sua efetivação na prática vai muito além da teoria, permitindo resultados satisfatórios, práticos e gratificantes para a enfermagem.

Recomenda-se maior envolvimento da instituição, promovendo, desta forma, recursos essenciais para a efetivação da SAE, além do investimento na capacitação dos enfermeiros, e assim, contribuirem para a conquista de benefícios próprios para a instituição, por meio de assistência de enfermagem qualificada e competente.

É imprescindível que os enfermeiros estejam imbuídos da ideologia da SAE, caso contrário, essa estratégia limitar-se-á a uma mera obrigação e terá como resultados insucessos e insatisfaçôes.

\section{Referências Bibliográficas}

Benedet, S. A., Gelbcke, F. L., Amante, L. N., Padilha, M. I., \& Pires, D. P. (2016). Processo de enfermagem: Instrumento da sistematização da assistência de enfermagem na percepção dos enfermeiros. Revista Online de Pesquisa Cuidado é Fundamental, 8(3), 4780-4788. doi: 10.9789/2175-5361.2016. v8i3.4780-4788

Carvalho, F. S., \& Barcelos, K. L. (2017). Sistematização da assistência de enfermagem: Vivências e desafios de enfermeiros de uma unidade de terapia intensiva adulto. Revista Brasileira de Ciências da Vida, 5(2), 21-25. Recuperado de http://jornal.faculdadecienciasdavida. com.br/index.php/RBCV/article/view/170/81 
Cavalcante, R. B., Otoni, A., Bernardes, M. F., Cunha, S. G., Santos, C. S., \& Silva, P., C. (2011). Experiências de sistematizaçáo da assistência de enfermagem no Brasil: Um estudo bibliográfico. Revista Enfermagem da UFSM, 1(3), 461-471. doi: $10.5902 / 217976922832$

Chaves, R. R., Silva, C. F., Motta, E., Ribeiro, E. D., \& Andrade, Y, N. (2016). Sistematização da assistência de enfermagem: Visão geral dos enfermeiros. Revista Enfermagem UFPE On Line, 10(4), 1280-1285. Recuperado de https://periodicos.ufpe.br/revistas/ revistaenfermagem/article/view/11114/12589

Cogo, E., Gehlen, M. H., Ilha, S., Zamberlan, C., Freitas, H. M., \& Backes, D. S. (2012). Sistematização da assistência de enfermagem no cenário hospitalar: Percepção dos enfermeiros. Cogitare Enfermagem, 17(3), 513-518. doi: 10.5380/ce.v17i3.29293

Garcia, T. R. (2016). Sistematizaçấo da assistência de enfermagem: Aspecto substantivo da prática profissional. Escola Anna Nery, 20(1), 5-10. doi: 10.5935/1414-8145.20160001

Lefèvre, F., \& Lefevre, A. M. (2010). Pesquisa de representação social: $O$ enfoque qualiquantitativo: Metodologia do discurso do sujeito coletivo. Brasília, Brasil: Liber Livro.

Massaroli, R., Martini, J. G., Massaroli, A., Lazzari, D. D., Oliveira, S. N., \& Canever Pedroso, B. P. (2015). Trabalho de enfermagem em unidade de terapia intensiva e sua interface com a sistematizaçáo da assistência. Escola Anna Nery, 19(2), 252-258. doi: 10.5935/1414-8145.20150033

Medeiros, A. L., Santos, S. R., \& Cabral, R. W. (2012). Sistematização da assistência de enfermagem na perspectiva dos enfermeiros: Uma abordagem metodológica na teoria fundamentada. Revista Gaúcha de Enfermagem, 33(3), 174-181. doi: 10.1590/ S1983-14472012000300023

Menezes, S. R., Priel, M. R., \& Pereira, L. L. (2011). Nurses' autonomy and vulnerability in the Nursing Assistance Systematization practice. Revista da Escola de Enfermagem da USP, 45(4), 953-958. doi: 10.1590/S0080-62342011000400023

Monteiro, A. K., Monteiro, A. K., Araújo, P. R., Gouveia, M. T., \& Alencar, A. A. (2013). Relato de experiência: Implantação da prescrição de enfermagem em uma unidade de terapia intensiva. Revista Interdisciplinar, 6(3), 174-177. Recuperado de https://revistainterdisciplinar.uninovafapi.edu.br/ index.php/revinter/article/view/24/pdf_50

Moreira, V., Santos, C. S., Oliveira, J. C., Reis, L. A., \&
Lima, E. F. (2013). Sistematização da assistência de enfermagem: Desafios na sua implantação. Revista InterScientia, 1(3), 60-79. Recuperado de https:// periodicos.unipe.br/index.php/interscientia/article/view/48/45

Nery, I. S., Santos, A. G., \& Sampaio, M. R. (2013). Dificuldades para a implantação sistematização da assistência de enfermagem em maternidades. Enfermagem em Foco, 4(1), 11-14. Recuperado de http:// revista.portalcofen.gov.br/index.php/enfermagem/ article/viewFile/494/184

Oliveira, A. P., Coelho, M. E., Almeida, V. C., Lisboa, K. W., \& Macêdo, A. L. (2012). Sistematização da assistência de enfermagem: Implementação em uma unidade de terapia intensiva. Revista Rene, 13(3), 601-612. Recuperado de http://www.revistarene.ufc. br/revista/index.php/revista/article/view/727/pdf

Pains, K. C, Oliveira, L. P., Moreira, M. T, \& Malta, T. R. (2009). Sistematização da assistência de enfermagem na prevenção de úlceras por pressão: Uma revisão literária. Recuperado de http://www.pergamum. univale.br/pergamum/tcc/Sistematizacaodaassistenciadeenfermagemnaprevencaodeulcerasporpressaoumarevisaoliteraria.pdf

Santos, Y. C., \& Silva, M. C. (2013). Implementation of the systematization of nursing care in a health institution: Experience report. Revista Enfermagem da UFPI, 2(4), 88-91. doi: 10.26694/reufpi. v2i4.1094

Silva, E. G., Oliveira, V. C., Neves, G. B., \& Guimarães, T. M. (2011). Nurses' knowledge about Nursing Care Systematization: From theory to practice. Revista da Escola de Enfermagem da USP, 45(6), 13801386. doi: 10.1590/S0080-62342011000600015

Silva, F. S., \& Carvalho Filha, F. S. (2017). Sistematização da assistência de enfermagem a pacientes dialíticos: Dificuldades, desafios e perspectivas. Arquivos de Ciências da Saúde, 24(2), 33-37. Recuperado de http://www.cienciasdasaude.famerp.br/index.php/ racs/article/view/599/687

Truppel, T. C., Meier, M. J., Calixto, R. C., Peruzzo, S. A., \& Crozeta, K. (2009). Sistematização da assistência de enfermagem em unidade de terapia intensiva. Revista Brasileira de Enfermagem, 62(2), 221227. doi: 10.1590/S0034-71672009000200008

Varela, G. C., \& Fernandes, S. C. (2013). Conhecimentos e práticas sobre a sistematização da assistência de enfermagem na estratégia saúde da família. Cogitare Enfermagem, 18(1), 124-130. doi: 10.5380/ ce.v18i1.31317 